\title{
Optimal Capital and Risk Allocations for Law- and Cash-Invariant Convex Functions*
}

\author{
Damir Filipović ${ }^{\dagger} \quad$ Gregor Svindland ${ }^{\ddagger}$
}

4 March 2008

\begin{abstract}
In this paper we provide the complete solution to the existence and characterization problem of optimal capital and risk allocations for not necessarily monotone, law-invariant convex risk measures on the model space $L^{p}$, for any $p \in[1, \infty]$. Our main result says that the capital and risk allocation problem always admits a solution via contracts whose payoffs are defined as increasing Lipschitz continuous functions of the aggregate risk.
\end{abstract}

Key words: exact convolutions, law-invariant risk measures, optimal capital and risk allocations.

\section{Introduction}

The problem of optimal capital and risk allocation among economic agents, or business units, has played a predominant role in the respective academic and industrial research areas for decades. The introduction of coherent and convex risk measures by Artzner et al. [3], Föllmer and Schied [19], and Frittelli and Rosazza-Gianin [20], respectively, has drawn the attention to study this problem using a new kind of approach (see Barrieu and El Karoui [5], Jouini et al. [21], Filipović and Kupper [14], Burgert and Rüschendorf [7], Acciaio [1], Ludkovski and Rüschendorf [24]). For some overview of the vast related finance literature we refer to Dana and Scarsini [11], Burgert and Rüschendorf [7] and the references therein.

*Filipović is supported by WWTF (Vienna Science and Technology Fund). Svindland gratefully acknowledges financial support from Munich Re Grant for doctoral students, and hospitality of the Research Unit of Financial and Actuarial Mathematics, Vienna University of Technology. We thank Beatrice Acciaio and Walter Schachermayer for fruitful discussions, and an anonymous referee for helpful remarks.

${ }^{\dagger}$ Vienna Institute of Finance, University of Vienna, and Vienna University of Economics and Business Administration, Heiligenstädter Strasse 46-48, A-1190 Wien, Austria. Email: damir.filipovic@vif.ac.at

${ }^{\ddagger}$ Mathematics Institute, University of Munich, Theresienstrasse 39, D-80333 München, Germany. Email: svindla@mathematik.uni-muenchen.de 
In this paper we provide the complete solution to the existence and characterization problem of optimal capital and risk allocations for not necessarily monotone, law-invariant convex risk measures on the model space $L^{p}$, for any $p \in[1, \infty]$. That is, we consider $n$ agents, or business units, with initial endowments $X_{1}, \ldots, X_{n} \in L^{p}$, who assess the riskiness of their positions by means of some not necessarily monotone, law-invariant convex risk measures $\rho_{i}$ on $L^{p}$. In order to minimize total and individual risk, the agents redistribute the aggregate endowment $X=X_{1}+\cdots+X_{n}$ among themselves. An optimal capital and risk allocation $Y_{1}, \ldots, Y_{n}$ satisfies $Y_{1}+\cdots+Y_{n}=X$ and

$$
\rho_{1}\left(Y_{1}\right)+\cdots+\rho_{n}\left(Y_{n}\right)=\inf _{\sum_{i=1}^{n} Z_{i}=X}\left(\rho_{1}\left(Z_{1}\right)+\cdots+\rho_{n}\left(Z_{n}\right)\right)
$$

As is often the case in practice, this redistribution procedure may be subject to frictions (e.g. limited fungibility of capital, see $[15,16]$ ) in the sense that not every allocation of $X$ is admissible. This can be formalized by restricting the risk measures $\rho_{i}$ accordingly, as proposed in [14], see also section 7 below. The restricted $\rho_{i}$ are typically not monotone, even though the original $\rho_{i}$ may be so. But this goes well with our framework, since we do not require monotonicity of $\rho_{i}$ right from the start. Examples for $\rho_{i}$ are mean-risk type risk measures, such as mean-variance, which obviously are convex law- and cash-invariant, but not monotone on $L^{p}$.

Our main result says that there always exists a solution to (1.1) in $L^{p}$ of the form $Y_{i}=f_{i}(X)$, for some increasing Lipschitz continuous functions $f_{i}: \mathbb{R} \rightarrow \mathbb{R}$ with $\sum_{i=1}^{n} f_{i}=\operatorname{Id}_{\mathbb{R}}$. In other words, the capital and risk allocation problem (1.1) always admits a solution via contracts whose payoffs are defined as (increasing Lipschitz continuous) functions of the aggregate risk $X$. This extremely useful fact is often assumed in economic contract theory. We now set this prevalent economic assumption on a sound mathematical basis.

As regards the uniqueness of the optimal allocation, one has always the freedom to rebalance the cash (section 3). We will show that some kind of strict convexity of $\rho_{1}$ is a sufficient condition for uniqueness of the optimal allocation up to rebalancing the cash. However, we also provide an example which illustrates that this condition is not necessary.

The existence proof is constructive. Following along the lines of Landsberger and Meilijson [25], we approximate the optimal allocation by simple random variables. At each level of approximation, the respective approximate solution is comonotone and optimal with respect to the approximate aggregate endowment. This allows, in principle, to compute the approximate optimal capital and risk allocation at any given level of accuracy. A useful fact, that will be explored elsewhere.

The article of Jouini et al. [21] has been most influential for this paper. Indeed, Jouini et al. [21] provide existence of optimal allocations for monotone law-invariant convex risk measures $\rho_{i}$ on $L^{\infty}$. Our motivation was to understand and extend their results beyond $L^{\infty}$, which from an applied perspective is a very limited model space (e.g. $L^{\infty}$ does not contain normal distributed random variables). Moreover, in view of the predominant use of mean-variance risk 
preferences in the literature and also the framework in [14], it was necessary to abandon the monotonicity assumption. Acciaio [1] provides further examples of this kind.

The remainder of the paper is as follows. In section 2, we state our main existence result (theorem 2.5) and illustrate it with various examples. In section 3 , we elaborate on the uniqueness of optimal allocations. In section 4 , we reduce the $n$-agent problem on $L^{p}$ to the case $n=2$ and $p=1$. Section 5 contains the core of the proof of theorem 2.5. This is a result of Landsberger and Meilijson [25], which however they only proved for simple random variables. We thus provide a full and comprehensive proof. In section 6 , we accomplish the proof of theorem 2.5. Section 7 discusses the above mentioned optimal allocation problem under constraints. Sections A and B contain lemmas that are needed for the proof of our main results.

\section{Existence of Optimal Allocations}

Throughout this paper $(\Omega, \mathcal{F}, \mathbb{P})$ denotes an atom-less standard probability space. All equalities and inequalities between random variables are understood in the $\mathbb{P}$-almost sure (a.s.) sense. If not specified otherwise, in the sequel, we let $p \in[1, \infty]$, and write $L^{p}=L^{p}(\Omega, \mathcal{F}, \mathbb{P})$ and $\|\cdot\|_{p}=\|\cdot\|_{L^{p}}$. The topological dual space of $L^{p}$ is denoted by $\left(L^{p}\right)^{*}$. It is well known that $\left(L^{p}\right)^{*}=L^{q}$ with $q=\frac{p}{p-1}$ for $p<\infty$, and that $\left(L^{\infty}\right)^{*} \supset L^{1}$ can be identified with $b a$, the space of all bounded finitely additive signed measures on $(\Omega, \mathcal{F})$ which vanish on $\mathbb{P}$-null sets. With some facilitating abuse of notation, we shall write $(X, Z) \mapsto E[X Z]$ for the dual pairing on $\left(L^{p},\left(L^{p}\right)^{*}\right)$ also for the case $p=\infty$.

We suppose the reader is familiar with standard terminology and basic duality theory for convex functions as outlined in [26] or [13]. We call a function $F: L^{p} \rightarrow[-\infty, \infty]$

(i) convex if $F(\lambda X+(1-\lambda) Y) \leq \lambda F(X)+(1-\lambda) F(Y)$ for all $\lambda \in[0,1]$ (here $\infty-\infty:=\infty)$,

(ii) proper if $F>-\infty$ and the (effective) domain dom $F:=\{F<\infty\} \neq \emptyset$,

(iii) cash-invariant if $F(0) \in \mathbb{R}$ and $F(X+m)=F(X)-m$ for all $m \in \mathbb{R}$,

(iv) monotone if $X \geq Y$ implies $F(X) \leq F(Y)$,

(v) positively homogeneous if $F(t X)=t F(X)$ for all $t \geq 0$,

(vi) law-invariant if $F(X)=F(Y)$ for all identically distributed $X \sim Y$.

The conjugate function

$$
F^{*}(Z)=\sup _{X \in L^{p}}(E[Z X]-F(X))
$$


of $F$ is lower semi-continuous (l.s.c.) and convex on $\left(L^{p}\right)^{*}$. The Fenchel-Moreau theorem (proposition 4.1 in [13]) states that $F^{* *}=F$ if $F$ is l.s.c. proper convex. The indicator function of a set $\mathcal{C}$ is defined as

$$
\delta(X \mid \mathcal{C}):= \begin{cases}0, & X \in \mathcal{C} \\ +\infty, & X \notin \mathcal{C}\end{cases}
$$

Definition 2.1. A convex risk measure on $L^{p}$ is a monotone convex cashinvariant function $L^{p} \rightarrow(-\infty, \infty]$. A coherent risk measure is a convex risk measure which in addition is positively homogeneous.

Convex risk measures on $L^{p}$ and more general model spaces have been studied, among others, in $[6,8,18,23]$. Let $\rho: L^{p} \rightarrow(-\infty, \infty]$ be a convex cashinvariant function. The set of all acceptable positions with respect to $\rho$ is $\mathcal{A}_{\rho}:=\left\{X \in L^{p} \mid \rho(X) \leq 0\right\}$. Note that, by cash-invariance, $\rho$ is l.s.c. if and only if its acceptance set $\mathcal{A}_{\rho}$ is closed.

Prominent examples of convex risk measures on $L^{p}$ are

(i) Average Value at Risk at level $\alpha \in[0,1]$, defined as

$$
\operatorname{AVaR}_{\alpha}(X):= \begin{cases}-\frac{1}{\alpha} \int_{0}^{\alpha} q_{X}(s) d s, & \alpha>0 \\ -\operatorname{essinf}(X), & \alpha=0\end{cases}
$$

where $q_{X}$ denotes the left-continuous quantile function of $X$, in particular, $\operatorname{AVaR}_{1}(X)=E[-X]$,

(ii) the entropic risk measure with parameter $\beta>0$, defined as

$$
\operatorname{Entr}_{\beta}(X)=\frac{1}{\beta} \log E\left[e^{-\beta X}\right]
$$

It is well known, and can be found in e.g. [18], that $\mathrm{AVaR}_{\alpha}$ is continuous on $L^{p}$ for $\alpha>0$, and -essinf and $\operatorname{Entr}_{\beta}$ are l.s.c. on $L^{p}$ and continuous on $L^{\infty}$.

We now formalize the above capital and risk allocation problem. Let $n \geq 2$ and $F_{1}, \ldots, F_{n}: L^{p} \rightarrow(-\infty, \infty]$ some proper convex functions. Their (infimal) convolution at $X \in L^{p}$ is defined as

$$
\square_{i=1}^{n} F_{i}(X)=F_{1} \square \ldots \square F_{n}(X):=\quad \inf _{\substack{X_{1}, \ldots, X_{n} \in L^{p} \\ \sum_{i=1}^{n} X_{i}=X}} \sum_{i=1}^{n} F_{i}\left(X_{i}\right) .
$$

The following properties are well known (see e.g. [26]).

Lemma 2.2. (i) $\square_{i=1}^{n} F_{i}: L^{p} \rightarrow[-\infty, \infty]$ is a convex function,

(ii) $\operatorname{dom} \square_{i=1}^{n} F_{i}=\sum_{i=1}^{n} \operatorname{dom} F_{i}$,

(iii) $\left(\square_{i=1}^{n} F_{i}\right)^{*}=\sum_{i=1}^{n} F_{i}^{*}$, 
(iv) $\operatorname{dom}\left(\square_{i=1}^{n} F_{i}\right)^{*}=\bigcap_{i=1}^{n} \operatorname{dom} F_{i}^{*}$.

Definition 2.3. Let $X \in L^{p}$. An n-tuple $\left(X_{1}, X_{2}, \ldots, X_{n}\right) \in L^{p} \times \ldots \times L^{p}$ such that $\sum_{i=1}^{n} X_{i}=X$ is called an allocation of $X$. The convolution $\square_{i=1}^{n} F_{i}$ is said to be exact at $X$ if there exists an allocation $\left(X_{1}, X_{2}, \ldots, X_{n}\right)$ of $X$ such that $\square_{i=1}^{n} F_{i}(X)=\sum_{i=1}^{n} F_{i}\left(X_{i}\right)$. Such a minimizing allocation is called an optimal allocation of $X$. The convolution is said to be exact if it is exact at every point $X \in L^{p}$.

Hence, the capital and risk allocation problem outlined in section 1 is equivalent to finding an optimal allocation for the convolution $\square_{i=1}^{n} \rho_{i}$ of $n$ convex cash-invariant functions $\rho_{i}$.

Definition 2.4. An allocation $\left(X_{1}, \ldots, X_{n}\right)$ of $X \in L^{p}$ is called comonotone if there exist increasing functions $f_{1}, \ldots, f_{n}: \mathbb{R} \rightarrow \mathbb{R}$ such that $\sum_{i=1}^{n} f_{i}=\operatorname{Id}_{\mathbb{R}}$ and $X_{i}=f_{i}(X)$ for all $i$. These functions $f_{i}$ are obviously 1-Lipschitz-continuous.

The following existence theorem is the main result of this paper. The proof is given in section 6 .

Theorem 2.5. Let $\rho_{1}, \ldots, \rho_{n}: L^{p} \rightarrow(-\infty, \infty]$ be l.s.c. law-invariant convex cash-invariant functions. Then $\square_{i=1}^{n} \rho_{i}$ is a l.s.c. law-invariant convex cashinvariant function on $L^{p}$. Moreover, for every $X \in L^{p}$ there exists a comonotone allocation $\left(X_{1}, \ldots, X_{n}\right)$ such that

$$
\square_{i=1}^{n} \rho_{i}(X)=\sum_{i=1}^{n} \rho_{i}\left(X_{i}\right) .
$$

In other words, $\square_{i=1}^{n} \rho_{i}$ is exact, and amongst the optimal allocations of any $X \in L^{p}$ there is always a comonotone one.

Remark 2.6. The economic message of theorem 2.5 is that the capital and risk allocation problem (1.1) always admits a solution via contracts whose payoffs are defined as (increasing Lipschitz continuous) functions $f_{i}(X)$ of the aggregate risk $X$. We note that this extremely useful fact is often assumed in economic contract theory. Theorem 2.5 now sets this prevalent economic assumption on a sound mathematical basis.

We note that the functions $\rho_{i}$ in theorem 2.5 do not have to be monotone. In case at least one of them is monotone (i.e. a convex risk measure), we may draw the following stronger conclusion:

Corollary 2.7. Let $\rho_{1}, \ldots, \rho_{n}: L^{p} \rightarrow(-\infty, \infty]$ be l.s.c. law-invariant convex cash-invariant functions, of which at least one is a convex risk measure. Then, $\square_{i=1}^{n} \rho_{i}$ is a l.s.c. law-invariant convex risk measure on $L^{p}$. Moreover, for every $X \in L^{p}$ there exists a comonotone optimal allocation.

Proof. In view of theorem 2.5 it remains to prove that $\square_{i=1}^{n} \rho_{i}$ is monotone. But this follows immediately from lemma 2.2 and the fact that a l.s.c. proper convex function $F: L^{p} \rightarrow(-\infty, \infty]$ is monotone if and only if dom $F^{*} \subset\left(L^{p}\right)_{-}^{*}$ (see e.g. [17] lemma 3.2). 
Next we apply theorem 2.5 to calculate optimal allocations for Average Value at Risks and entropic risk measures, respectively. These convolutions are discussed thoroughly in e.g. [4] or [22] on $L^{\infty}$. In contrast, we provide our results on $L^{1}$.

Example 2.8. The Average Value at Risk (2.2) can be represented as

$$
\operatorname{AVaR}_{\alpha}(X)=\sup \left\{E_{\mathbb{Q}}[-X] \mid \mathbb{Q} \ll \mathbb{P}, \frac{d \mathbb{Q}}{d \mathbb{P}} \leq \frac{1}{\alpha}\right\} .
$$

This representation is well known for $X \in L^{\infty}$ (see e.g. [19]) and is extended to $L^{1}$ in [18]. Now let $0 \leq \beta \leq \gamma \leq 1$, then

$$
\mathrm{AVaR}_{\beta} \square \mathrm{AVaR}_{\gamma}=\mathrm{AVaR}_{\gamma} .
$$

This is easily verified employing theorem 2.5, lemma 2.2, the Fenchel-Moreau theorem and the fact that dom $\mathrm{AVaR}_{\gamma}^{*} \subset \operatorname{dom} \mathrm{AVaR}_{\beta}^{*}$, implied by (2.5).

Example 2.9. The entropic risk measure (2.3) can be represented as

$$
\operatorname{Entr}_{\beta}(X)=\sup _{\mathbb{Q} \in \mathcal{M}(\mathbb{P})} E_{\mathbb{Q}}[-X]-\frac{1}{\beta} H(\mathbb{Q} \mid \mathbb{P})
$$

where $H(\mathbb{Q} \mid \mathbb{P})=E_{\mathbb{Q}}\left[\log \frac{d \mathbb{Q}}{d \mathbb{P}}\right]$ denotes the relative entropy and $\mathcal{M}(\mathbb{P})$ is the set of all probability measures $\mathbb{Q}$ on $(\Omega, \mathcal{F})$ such that $\mathbb{Q} \ll \mathbb{P}$ and $d \mathbb{Q} / d \mathbb{P}$ is bounded. This representation is well known for $X \in L^{\infty}$ (see e.g. [19]) and is extended to $L^{1}$ in [18]. Now let $0<\beta \leq \gamma$. Theorem 2.5 and lemma 2.2 justify the following dual approach, for any $X \in L^{1}$ :

$$
\begin{aligned}
\operatorname{Entr}_{\beta} \square \operatorname{Entr}_{\gamma}(X) & =\sup _{\mathbb{Q} \in \mathcal{M}(\mathbb{P})} E_{\mathbb{Q}}[-X]-\frac{1}{\beta} H(\mathbb{Q} \mid \mathbb{P})-\frac{1}{\gamma} H(\mathbb{Q} \mid \mathbb{P}) \\
& =\sup _{\mathbb{Q} \in \mathcal{M}(\mathbb{P})} E_{\mathbb{Q}}[-X]-\frac{\beta+\gamma}{\beta \gamma} H(\mathbb{Q} \mid \mathbb{P}) \\
& =\operatorname{Entr}_{\frac{\beta \gamma}{\beta+\gamma}}(X) .
\end{aligned}
$$

Now, in the search for comonotone optimal allocations, the following ansatz seems natural. We guess that for any $X \in L^{1}$ there must be an (obviously comonotone) optimal allocation amongst the allocations of type $(a X, b X)$ where $a \in[0,1]$ and $b:=1-a$. If so, then

$$
\frac{\beta+\gamma}{\beta \gamma} \log E\left[e^{-\frac{\beta \gamma}{\beta+\gamma} X}\right]=\frac{1}{\beta} \log E\left[e^{-\beta a X}\right]+\frac{1}{\gamma} \log E\left[e^{-\gamma b X}\right]
$$

which is equivalent to

$$
\log E\left[e^{-\frac{\beta \gamma}{\beta+\gamma} X}\right]=\frac{\gamma}{\beta+\gamma} \log E\left[e^{-\beta a X}\right]+\frac{\beta}{\beta+\gamma} \log E\left[e^{-\gamma b X}\right] .
$$

Clearly, $a=\frac{\gamma}{\beta+\gamma}$ and $b=\frac{\beta}{\beta+\gamma}$ satisfy this equation. Hence, $\left(\frac{\gamma}{\beta+\gamma} X, \frac{\beta}{\beta+\gamma} X\right)$ is a comonotone optimal allocation of $X$. 
Remark 2.10. The l.s.c. requirement in theorem 2.5 cannot be dropped, as the following example shows. Let $\rho_{1}(X)=-E[X]+\delta\left(X^{-} \mid L^{\infty}\right)$, and $\rho_{2}=\operatorname{AVaR}_{\alpha}$ on $L^{1}$, for some $\alpha \in(0,1)$. Clearly, the acceptance set $\mathcal{A}_{\rho_{1}}$ of $\rho_{1}$ is not closed and thus $\rho_{1}$ is not l.s.c. on $L^{1}$. We claim that

$$
\rho_{1} \square \rho_{2}=-E .
$$

Indeed, on the one hand, we know that $\rho_{1}^{*}=\delta(\cdot \mid\{-1\})$ and that $\rho_{2}^{*}(-1)=0$ (see (A.17) below). Hence, $\left(\rho_{1} \square \rho_{2}\right)^{*}=\rho_{1}^{*}+\rho_{2}^{*}=\delta(\cdot \mid\{-1\})$ which implies $\rho_{1} \square \rho_{2} \geq-E$. On the other hand,

$$
\begin{aligned}
\rho_{1} \square \rho_{2}(X) & =\inf _{X_{1}+X_{2}=X} E\left[-X_{1}\right]+\delta\left(X_{1}^{-} \mid L^{\infty}\right)+\operatorname{AVaR}_{\alpha}\left(X_{2}\right) \\
& \leq \inf _{K \in \mathbb{N}} E\left[-X 1_{\{X>-K\}}\right]+\operatorname{AVaR}_{\alpha}\left(X 1_{\{X \leq-K\}}\right) \\
& \leq E[-X]+\lim _{K \rightarrow \infty} \operatorname{AVaR}_{\alpha}\left(X 1_{\{X \leq-K\}}\right)=E[-X]
\end{aligned}
$$

because $\mathrm{AVaR}_{\alpha}$ is continuous and $X 1_{\{X \leq-K\}} \rightarrow 0$ in $L^{1}$ for $K \rightarrow \infty$. This proves (2.7).

Now, choose any $X \in L^{1}$ being unbounded from below. Suppose there is an optimal allocation $\left(X_{1}, X_{2}\right)$ of $X$. Then $X_{1}$ must be bounded and $X_{2}$ unbounded from below, respectively. In view of lemma 2.11 below, we thus have $\operatorname{AVaR}_{\alpha}\left(X_{2}\right)>E\left[-X_{2}\right]$, and hence

$$
\begin{aligned}
\rho_{1} \square \rho_{2}(X) & =\rho_{1}\left(X_{1}\right)+\rho_{2}\left(X_{2}\right) \\
& =E\left[-X_{1}\right]+\delta\left(X_{1}^{-} \mid L^{\infty}\right)+\operatorname{AVaR}_{\alpha}\left(X_{2}\right) \\
& >E\left[-X_{1}\right]+E\left[-X_{2}\right]=E[-X],
\end{aligned}
$$

which contradicts (2.7). Hence, there exists no optimal allocation of $X$.

Lemma 2.11. Let $0 \leq \beta<\gamma \leq 1$. Then

$$
\operatorname{AVaR}_{\beta}(X) \geq \operatorname{AVaR}_{\gamma}(X),
$$

and equality holds if and only if $X \geq c$ a.s. and $\mathbb{P}[X=c] \geq \gamma$ for some constant $c \in \mathbb{R}$. In particular, $\operatorname{AVaR}_{\beta}(X)=E[-X]$ if and only if $X$ is constant.

Proof. The case $\beta=0$ is obvious. Suppose $\beta>0$. Since $q_{X}$ is increasing, we obviously have

$$
(\gamma-\beta) \int_{0}^{\beta} q_{X}(s) d s \leq \beta \int_{\beta}^{\gamma} q_{X}(s) d s,
$$

with equality if and only if $q_{X}(s)=q_{X}(\gamma)$ for all $s \leq \gamma$. This proves the claim.

Remark 2.12. Also the law-invariance requirement in theorem 2.5 cannot be dropped: let $Z \geq 0$ non-constant with $E[Z]=1$. Then $\rho_{1}=-E$ and $\rho_{2}=$ $E[-Z \cdot]$ are convex risk measures on $L^{\infty}$ and $\rho_{2}$ is not law-invariant. Thus theorem 2.5 does not apply. Indeed, the convolution $\rho_{1} \square \rho_{2} \equiv-\infty$ is not exact.\| 


\section{Uniqueness of Optimal Allocations}

Let $\rho_{1}, \ldots, \rho_{n}: L^{p} \rightarrow(-\infty, \infty]$ be convex cash-invariant functions. Due to cash-invariance of $\rho_{i}$, uniqueness of an optimal allocation can only hold up to rebalancing the cash. That is, $\left(X_{1}, \ldots, X_{n}\right)$ is an optimal allocation of $X$ if and only if $\left(X_{1}+c_{1}, \ldots, X_{n}+c_{n}\right)$ is so, for all cash positions $c_{i} \in \mathbb{R}$ with $\sum_{i=1}^{n} c_{i}=0$.

The following sufficient condition for uniqueness is straightforward.

Proposition 3.1. Suppose $\rho_{1}, \ldots, \rho_{n-1}$ are strictly convex in the sense that

$$
\rho_{i}(\lambda X+(1-\lambda) Y)<\lambda \rho_{i}(X)+(1-\lambda) \rho_{i}(Y) \text { for all } \lambda \in(0,1),
$$

for all $X, Y \in \operatorname{dom} \rho_{i}$ with $X-Y \notin \mathbb{R}$. Then any optimal allocation of $X \in L^{p}$ with $\square_{i=1}^{n} \rho_{i}(X)<\infty$ is unique up to rebalancing the cash.

Proof. Let $X \in L^{p}$ with $\square_{i=1}^{n} \rho_{i}(X)<\infty$. We argue by contradiction and suppose $\left(X_{1}, \ldots, X_{n}\right)$ and $\left(Y_{1}, \ldots, Y_{n}\right)$ are optimal allocations of $X$ with $X_{j}-$ $Y_{j} \notin \mathbb{R}$ for some $1 \leq j \leq n-1$. Then, for any $\lambda \in(0,1), Z_{i}=\lambda X_{i}+(1-\lambda) Y_{i}$ is an allocation of $X$ with

$$
\sum_{i=1}^{n} \rho_{i}\left(Z_{i}\right)<\lambda \sum_{i=1}^{n} \rho_{i}\left(X_{i}\right)+(1-\lambda) \sum_{i=1}^{n} \rho_{i}\left(Y_{i}\right)=\square_{i=1}^{n} \rho_{i}(X) .
$$

But this contradicts the optimality of $X_{i}$, whence the claim.

For instance, the optimal allocation for the entropic risk measure in example 2.9 is unique up to rebalancing the cash. More recent examples of strictly convex risk measures can be found in [10].

Without the strict convexity assumption in proposition 3.1, uniqueness does not hold in general. A trivial example is

$$
(-E) \square(-E)=-E \text {. }
$$

In this case, all allocations of any $X \in L^{1}$ are optimal allocations of $X$.

Moreover, the following example shows that uniqueness may even fail in the class of comonotone allocations without the strict convexity assumption in proposition 3.1.

Example 3.2. Let $0 \leq \beta \leq \gamma<1$. In view of (2.5), there exists some $A \in \mathcal{F}$ with $\mathbb{P}[A]>0$ and some $\bar{Q} \ll \mathbb{P}$ with $d \mathbb{Q} / d \mathbb{P} \leq 1 / \gamma \leq 1 / \beta$ and $\mathbb{Q}[A]=0$. Consequently,

$$
0=\operatorname{AVaR}_{\beta}\left(1_{A}\right)=\operatorname{AVaR}_{\gamma}\left(1_{A}\right)=\operatorname{AVaR}_{\beta} \square \operatorname{AVaR}_{\gamma}\left(1_{A}\right) .
$$

Hence, both $\left(1_{A}, 0\right)$ and $\left(0,1_{A}\right)$ are comonotone optimal allocations of $1_{A}$. |

On the other hand, the strict convexity assumption in proposition 3.1 is not necessary for uniqueness up to rebalancing the cash. This is shown by the following example.

Example 3.3. Let $\beta \in(0,1)$. We know that $\mathrm{AVaR}_{\beta} \square-E=-E$ (example 2.8). Suppose $(Y, X-Y)$ is an optimal allocation of $X$. This implies $\operatorname{AVaR}_{\beta}(Y)+$ $E[-(X-Y)]=E[-X]$, that is, $\operatorname{AVaR}_{\beta}(Y)=E[-Y]$. In view of lemma 2.11 we conclude that $Y$ must be constant, i.e. a cash position. 


\section{Problem Reduction}

First note that it is enough to prove theorem 2.5 for $n=2$, the rest follows by induction. Indeed, if $\rho_{1} \square \cdots \square \rho_{n-1}>-\infty$ then associativity holds:

$$
\square_{i=1}^{n} \rho_{i}=\left(\square_{i=1}^{n-1} \rho_{i}\right) \square \rho_{n} .
$$

For the sake of simplicity, we will further restrict our studies to the case $p=1$. By nature of the arguments presented in the proof of theorem 2.5 (section 6), it will become clear that they all literally carry over to $L^{p}$, simply by replacing $L^{1}$ with $L^{p}$ and choosing the appropriate dual. However, in what follows, we give another justification for the retreat to $L^{1}$ by proving that the assertions of theorem 2.5 for the $L^{p}$-cases can be derived as a corollary from knowing it for the case of $L^{1}$. The main ingredient to this is the following result:

Theorem 4.1. Let $\rho: L^{p} \rightarrow(-\infty, \infty]$ be a l.s.c. law-invariant convex cashinvariant function. Then, there is a unique l.s.c. law-invariant convex cashinvariant function $\widetilde{\rho}: L^{1} \rightarrow(-\infty, \infty]$ such that $\rho=\left.\widetilde{\rho}\right|_{L^{p}}$.

Proof. see [18].

Let

$$
\mathbb{A}:=\left\{(f, g) \mid f, g: \mathbb{R} \rightarrow \mathbb{R} \text { are increasing }, f+g=\operatorname{Id}_{\mathbb{R}}\right\} .
$$

Clearly, if $(f, g) \in \mathbb{A}$, then both $f$ and $g$ are 1-Lipschitz-continuous. Hence, $|f(X)| \leq|X|+|f(0)|$ and $|g(X)| \leq|X|+|g(0)|$, implying that

$$
\text { if } X \in L^{p} \text { then }(f(X), g(X)) \in L^{p} \times L^{p} \text {. }
$$

Thus, for any $X \in L^{p}$ the set $\{(f(X), g(X)) \mid(f, g) \in \mathbb{A}\}$ is the set of all comonotone 2-dimensional allocations of $X$.

Corollary 4.2. Let $\rho_{1}, \rho_{2}: L^{p} \rightarrow(-\infty, \infty]$ be two l.s.c. law-invariant convex cash-invariant functions, and let $\widetilde{\rho}_{1}$ and $\widetilde{\rho}_{2}$ be the unique l.s.c. law-invariant convex cash-invariant functions on $L^{1}$ such that $\rho_{i}=\left.\widetilde{\rho}_{i}\right|_{L^{p}}, i=1,2$. Suppose the assertions of theorem 2.5 hold for $\widetilde{\rho}_{1} \square \widetilde{\rho}_{2}$. Then $\rho_{1} \square \rho_{2}=\left.\widetilde{\rho}_{1} \square \widetilde{\rho}_{2}\right|_{L^{p}}$. In particular, the assertions of theorem 2.5 are true for $\rho_{1} \square \rho_{2}$ too.

Proof. By assumption, for any $X \in L^{p} \subset L^{1}$ there is a comonotone allocation $(f(X), g(X))$ such that $\widetilde{\rho}_{1} \square \widetilde{\rho}_{2}(X)=\widetilde{\rho}_{1}(f(X))+\widetilde{\rho}_{2}(g(X))$. Clearly, $\widetilde{\rho}_{1} \square \widetilde{\rho}_{2}(X) \leq$ $\rho_{1} \square \rho_{2}(X)$, and since $(f(X), g(X)) \in L^{p} \times L^{p}$ by (4.9), we deduce that

$$
\widetilde{\rho}_{1} \square \widetilde{\rho}_{2}(X)=\rho_{1}(f(X))+\rho_{2}(g(X))=\rho_{1} \square \rho_{2}(X) .
$$

Hence, firstly, $\rho_{1} \square \rho_{2}$ is simply the restriction of $\widetilde{\rho}_{1} \square \widetilde{\rho}_{2}$ to $L^{p}$ and thus a l.s.c. (w.r.t. $\|\cdot\|_{p}$ ) law-invariant convex cash-invariant function. (The l.s.c. stems from the fact that $\|\cdot\|_{p}$-convergence implies $\|\cdot\|_{1}$-convergence.) Secondly, $\rho_{1} \square \rho_{2}$ is exact and there is always a comonotone optimal allocation. 


\section{Comonotone Concave Order Improvement}

We denote by $\succeq_{c}$ the concave order on $L^{1}$, that is, $X \succeq_{c} Y$ if and only if $E[u(X)] \geq E[u(Y)]$ for all concave functions $u: \mathbb{R} \rightarrow \mathbb{R}$. Clearly, since $\operatorname{Id}_{\mathbb{R}}$ and $-\operatorname{Id}_{\mathbb{R}}$ are concave functions, $X \succeq_{c} Y$ implies $E[X]=E[Y]$. Moreover,

$$
X \succeq_{c} Y \quad \Leftrightarrow \quad E[X]=E[Y] \text { and } E\left[(X-c)^{+}\right] \leq E\left[(Y-c)^{+}\right] \forall c \in \mathbb{R} .
$$

For a proof of (5.10), we refer to corollary 2.62 in [19].

Proposition 5.1 below will turn out to be the basis of the proof of theorem 2.5. It is based upon the results of Landsberger and Meilijson [25], and states that every allocation is dominated in concave order by a comonotone allocation. The importance of this results becomes clear by (A.14) where we establish that any l.s.c. law-invariant convex function is monotone w.r.t. the $\succeq_{c}$-order.

Proposition 5.1. (see proposition 1 in [25]) For any allocation $(Y, Z)$ of $X \in$ $L^{1}$, there is $(f, g) \in \mathbb{A}$ such that $f(X) \succeq_{c} Y$ and $g(X) \succeq_{c} Z$.

Unfortunately, Landsberger and Meilijson [25] only proved this result for random variables $X$ supported by a finite set. For sake of completeness, we thus give a full proof here.

Proof. We divide the proof into three steps.

Step 1: We start out as in [25] by noticing that Jensen's inequality implies that $(E[Y \mid X], E[Z \mid X])$ is an allocation of $X$ which is at least as good as $(Y, Z)$, meaning that $E[Y \mid X] \succeq_{c} Y$ and $E[Z \mid X] \succeq_{c} Z$. Let $h_{1}, h_{2}: \mathbb{R} \rightarrow \mathbb{R}$ be measurable functions such that $h_{1}(X)=E[Y \mid X], h_{2}(X)=E[Z \mid X]$. Clearly, we may assume that $h_{1}+h_{2}=\operatorname{Id}_{\mathbb{R}}$. If $h_{1}$ and $h_{2}$ are increasing, we are done, if not, we go on improving this allocation. However, we have now established that during the remainder of this proof we may restrict ourselves to improve allocations $(Y, Z)$ of type $Y=h_{1}(X)$ and $Z=h_{2}(X)$ for some measurable functions $h_{1}, h_{2}: \mathbb{R} \rightarrow \mathbb{R}$ such that $h_{1}+h_{2}=\operatorname{Id}_{\mathbb{R}}$.

Step 2: Suppose $X$ is a simple random variable, i.e. $X=\sum_{i=1}^{n} x_{i} 1_{A_{i}}$ for a partition $A_{1}, \ldots, A_{n}$ of $\Omega$ and real numbers $x_{i}$ such that $x_{i} \neq x_{j}$ for $i \neq j$. Let $y_{i}:=h_{1}\left(x_{i}\right)$ and $z_{i}:=h_{2}\left(x_{i}\right)$. Then $h_{1}(X)=\sum_{i=1}^{n} y_{i} 1_{A_{i}}$ and $h_{2}(X)=$ $\sum_{i=1}^{n} z_{i} 1_{A_{i}}$. We set $x:=\left(x_{1}, \ldots, x_{n}\right), y:=\left(y_{1}, \ldots, y_{n}\right), z:=\left(z_{1}, \ldots, z_{n}\right)$ and $p_{k}:=\mathbb{P}\left(A_{k}\right), k=1, \ldots, n$. Let $\pi$ be a permutation of $\{1, \ldots, n\}$ such that $x_{\pi}:=\left(x_{\pi(1)}, \ldots, x_{\pi(n)}\right) \in \mathcal{D}:=\left\{\widetilde{x} \in \mathbb{R}^{n} \mid \widetilde{x}_{1} \leq \widetilde{x}_{2} \leq \ldots \leq \widetilde{x}_{n}\right\}$. Observe that $\left(h_{1}(X), h_{2}(X)\right)$ is comonotone if and only if $y_{\pi}, z_{\pi} \in \mathcal{D}$. For sake of brevity we may and will assume w.l.o.g. that $x \in \mathcal{D}$ already. Supposing that $(y, z)$ is not comonotone, i.e. $y \notin \mathcal{D}$ or $z \notin \mathcal{D}$ or both, the following algorithm by $\mathrm{M}$. Landsberger and I. Meilijson transfers $(y, z)$ into a comonotone allocation:

Since $(y, z)$ is not comonotone, there must exist an $i$ such that $y_{1} \leq \ldots \leq y_{i}$, $z_{1} \leq \ldots \leq z_{i}$ but either $y_{i+1}<y_{i}$ or $z_{i+1}<z_{i}$. W.l.o.g. let us assume that $z_{i+1}<z_{i}$. Then there is a smallest $j$ such that $z_{i+1}<z_{j}$. For $k=j, \ldots, i$ we set

$$
y_{k}^{\text {new }}=y_{k}+\frac{p_{i+1}}{\sum_{l=j}^{i+1} p_{l}}\left(z_{j}-z_{i+1}\right) \quad \text { and } \quad z_{k}^{\text {new }}=z_{k}-\frac{p_{i+1}}{\sum_{l=j}^{i+1} p_{l}}\left(z_{j}-z_{i+1}\right)
$$


whereas

$$
y_{i+1}^{n e w}=y_{i+1}-\frac{\sum_{l=j}^{i} p_{l}}{\sum_{l=j}^{i+1} p_{l}}\left(z_{j}-z_{i+1}\right) \quad \text { and } \quad z_{i+1}^{n e w}=z_{i+1}+\frac{\sum_{l=j}^{i} p_{l}}{\sum_{l=j}^{i+1} p_{l}}\left(z_{j}-z_{i+1}\right) .
$$

The other coordinates of $y$ and $z$ are left unchanged. Finally, set $y:=y^{n e w}$ and $z:=z^{\text {new }}$ and repeat the procedure in case the output is not comonotone.

Let $\left(Y^{\text {new }}, Z^{\text {new }}\right):=\left(\sum_{i=1}^{n} y_{i}^{\text {new }} 1_{A_{i}}, \sum_{i=1}^{n} z_{i}^{\text {new }} 1_{A_{i}}\right)$. Firstly, $\left(Y^{\text {new }}, Z^{\text {new }}\right)$ is obviously an allocation of $X$, secondly, we claim that $Y^{\text {new }} \succeq_{c} Y$ and $Z^{\text {new }} \succeq_{c}$ $Z$, i.e. each cycle of the algorithm improves the allocation, and finally, it is easily verified that the algorithm returns a comonotone allocation in at most $n(n-1) / 2$ cycles (observe that $z_{j}^{\text {new }}=z_{i+1}^{\text {new }}$ ). In order to show that $Y^{\text {new }} \succeq_{c} Y$ and $Z^{\text {new }} \succeq_{c} Z$, let $u: \mathbb{R} \rightarrow \mathbb{R}$ be any concave function. Introducing the abbreviations

$$
\alpha:=\frac{p_{i+1}}{\sum_{l=j}^{i+1} p_{l}} \in(0,1) \quad \text { and } \quad \lambda_{k}:=\frac{z_{j}-z_{i+1}}{z_{k}-z_{i+1}} \in(0,1]
$$

and recalling that concavity is equivalent to

$$
\forall a<b<c: \frac{u(b)-u(a)}{b-a} \geq \frac{u(c)-u(a)}{c-a} \geq \frac{u(c)-u(b)}{c-b},
$$

we compute:

$$
\begin{aligned}
\sum_{k=j}^{i+1} u\left(z_{k}^{\text {new }}\right) p_{k}= & \sum_{k=j}^{i} u\left(\left(1-\alpha \lambda_{k}\right) z_{k}+\alpha \lambda_{k} z_{i+1}\right) p_{k}+u\left((1-\alpha) z_{j}+\alpha z_{i+1}\right) p_{i+1} \\
\geq & \sum_{k=j}^{i}\left[\left(1-\alpha \lambda_{k}\right) u\left(z_{k}\right)+\alpha \lambda_{k} u\left(z_{i+1}\right)\right] p_{k} \\
& +\left[(1-\alpha) u\left(z_{j}\right)+\alpha u\left(z_{i+1}\right)\right] p_{i+1} \\
= & \sum_{k=j}^{i+1} u\left(z_{k}\right) p_{k}+(1-\alpha)\left(u\left(z_{j}\right)-u\left(z_{i+1}\right)\right) p_{i+1} \\
& -\alpha \sum_{k=j}^{i} \lambda_{k}\left(u\left(z_{k}\right)-u\left(z_{i+1}\right)\right) p_{k} \\
& (5.11) \sum_{k=j}^{i+1} u\left(z_{k}\right) p_{k}
\end{aligned}
$$

because $\lambda_{k}\left(u\left(z_{k}\right)-u\left(z_{i+1}\right)\right) \leq u\left(z_{j}\right)-u\left(z_{i+1}\right)$ by inequality (5.11). A similar computation for $Y^{\text {new }}$ shows that $Y^{\text {new }} \succeq_{c} Y$ and $Z^{\text {new }} \succeq_{c} Z$.

Step 3: Let $X$ be any integrable random variable. Recalling the usual monotone approximation from Lebesgue integration theory, let $\left(Y_{n}\right)_{n \in \mathbb{N}}$ and $\left(Z_{n}\right)_{n \in \mathbb{N}}$ be sequences of simple random variables converging $\mathbb{P}$-a.s. and in $L^{1}$ to 
$Y$ and $Z$ respectively such that $\left|Y_{n}\right| \leq|Y|$ and $\left|Z_{n}\right| \leq|Z|$ for all $n \in \mathbb{N}$. Then $X_{n}:=Y_{n}+Z_{n}$ converges to $X \mathbb{P}$-a.s. and in $L^{1}$. By step 2, for each $n \in \mathbb{N}$, there exists a comonotone improvement $\left(f_{n}\left(X_{n}\right), g_{n}\left(X_{n}\right)\right)$ of $\left(Y_{n}, Z_{n}\right)$. Choose $N \in \mathbb{N}$ such that $\left\|Y_{n}\right\|_{1} \leq\|Y\|_{1}+1,\left\|Z_{n}\right\|_{1} \leq\|Z\|_{1}+1$, and $\left\|X_{n}\right\|_{1} \leq\|X\|_{1}+1$ for all $n \geq N$. Since all $f_{n}$ (and $g_{n}$ ) are 1-Lipschitz-continuous, we have that $\left|f_{n}(0)\right| \leq\left|X_{n}\right|+\left|f_{n}\left(X_{n}\right)\right|$. Taking expectations on both sides yields

$$
\left|f_{n}(0)\right| \leq E\left[\left|X_{n}\right|\right]+E\left[\left|f_{n}\left(X_{n}\right)\right|\right] \leq E\left[\left|X_{n}\right|\right]+E\left[\left|Y_{n}\right|\right],
$$

because $f_{n}\left(X_{n}\right) \succeq_{c} Y_{n}$ and $x \mapsto-|x|$ is concave. Hence, if $n \geq N$, we get $\left|f_{n}(0)\right| \leq\|X\|_{1}+\|Y\|_{1}+2=: K_{1}$ and similarly $\left|g_{n}(0)\right| \leq\|X\|_{1}+\|Z\|_{1}+$ $2=: K_{2}$, and thus $f_{n}(0), g_{n}(0) \in[-K, K]$ for $K:=\max \left\{K_{1}, K_{2}\right\}$. Therefore, by lemma B.1, there is a subsequence $\left(f_{n_{k}}\right)_{k \in \mathbb{N}}$ of $\left(f_{n}\right)_{n \in \mathbb{N}}$ and a 1-Lipschitzcontinuous increasing function $f: \mathbb{R} \rightarrow \mathbb{R}$ such that $f(a)=\lim _{k \rightarrow \infty} f_{n_{k}}(a), a \in$ $\mathbb{R}$. Now it is easily verified that $\left(g_{n_{k}}\right)_{k \in \mathbb{N}}$ converges pointwise to the 1 -Lipschitzcontinuous increasing function $g:=\mathrm{Id}_{\mathbb{R}}-f$. Hence, the sequence $f_{n_{k}}\left(X_{n_{k}}\right)$ converges $\mathbb{P}$-a.s. to $f(X)$, and $g_{n_{k}}\left(X_{n_{k}}\right)=X_{n_{k}}-f_{n_{k}}\left(X_{n_{k}}\right)$ converges $\mathbb{P}$-a.s. to $g(X)$. Since $\left|f_{n_{k}}\left(X_{n_{k}}\right)\right| \leq\left|X_{n_{k}}\right|+K \leq|Y|+|Z|+K$ and $\left|g_{n_{k}}\left(X_{n_{k}}\right)\right| \leq$ $|Y|+|Z|+K$ for large enough $k \in \mathbb{N}$, we can apply the dominated convergence theorem which yields $f(X), g(X) \in L^{1}$ and $\left\|f(X)-f_{n_{k}}\left(X_{n_{k}}\right)\right\|_{1} \rightarrow 0, \| g(X)-$ $g_{n_{k}}\left(X_{n_{k}}\right) \|_{1} \rightarrow 0$ for $k \rightarrow \infty$. Moreover, in view of (5.10), we have that

$$
E[f(X)]=\lim _{k \rightarrow \infty} E\left[f_{n_{k}}\left(X_{n_{k}}\right)\right]=\lim _{k \rightarrow \infty} E\left[Y_{n_{k}}\right]=E[Y],
$$

and for all $c \in \mathbb{R}$ :

$$
\begin{aligned}
E\left[(f(X)-c)^{+}\right] & =\lim _{k \rightarrow \infty} E\left[\left(f_{n_{k}}\left(X_{n_{k}}\right)-c\right)^{+}\right] \\
& \leq \lim _{k \rightarrow \infty} E\left[\left(Y_{n_{k}}-c\right)^{+}\right]=E\left[(Y-c)^{+}\right],
\end{aligned}
$$

and similarly for $g$. Hence, $(f(X), g(X))$ is a comonotone allocation of $X$ satisfying $f(X) \succeq_{c} Y$ and $g(X) \succeq_{c} Z$ according to (5.10).

\section{Proof of Theorem 2.5}

In view of section 4 we only have to prove theorem 2.5 for $n=2$ and $p=1$. To this end, let $\rho_{1}, \rho_{2}: L^{1} \rightarrow(-\infty, \infty]$ be l.s.c. law-invariant convex cash-invariant functions. We divide the proof into four steps.

Step 1: $\rho_{1} \square \rho_{2}$ is proper, convex, and cash-invariant.

Proof. It is easily verified that the convolution preserves the convexity of $\rho_{1}$ and $\rho_{2}$. By (A.16) we have that $\rho_{i}(X) \geq-E[X]+\rho_{i}(0)$ for all $X \in L^{1}$ and $i=1,2$. Hence,

$$
\begin{aligned}
\rho_{1} \square \rho_{2}(X) & =\inf _{X_{1}+X_{2}=X} \rho_{1}\left(X_{1}\right)+\rho_{2}\left(X_{2}\right) \\
& \geq \inf _{X_{1}+X_{2}=X}-E\left[X_{1}\right]-E\left[X_{2}\right]+\rho_{1}(0)+\rho_{2}(0) \\
& =-E[X]+\rho_{1}(0)+\rho_{2}(0),
\end{aligned}
$$


so $\rho_{1} \square \rho_{2}$ is proper and $\rho_{1} \square \rho_{2}(0)=\rho_{1}(0)+\rho_{2}(0)<\infty$. Furthermore, for all $r \in \mathbb{R}$ we get

$$
\rho_{1} \square \rho_{2}(X+r)=\inf _{Y \in L^{1}} \rho_{1}(X+r-Y)+\rho(Y)=\rho_{1} \square \rho_{2}(X)-r
$$

due to the cash-invariance of $\rho_{1}$.

Step 2: $\rho_{1} \square \rho_{2}(X)=\inf _{(f, g) \in \mathbb{A}} \rho_{1}(f(X))+\rho_{2}(g(X)), \quad X \in L^{1}$.

Proof. This is an immediate consequence of proposition 5.1 and (A.14).

Step 3: $\rho_{1} \square \rho_{2}$ is exact, and for each $X \in L^{1}$ there exists a comonotone optimal allocation.

Proof. Suppose $X \in L^{1}$ is such that $\rho_{1} \square \rho_{2}(X)=\infty$. Then every allocation $(f(X), g(X)),(f, g) \in \mathbb{A}$, is optimal.

Now let $X \in \operatorname{dom} \rho_{1} \square \rho_{2}$ and choose a sequence $\left(f_{n}, g_{n}\right) \in \mathbb{A}, n \in \mathbb{N}$, such that $\rho_{1} \square \rho_{2}(X)=\lim _{n \rightarrow \infty} \rho_{1}\left(f_{n}(X)\right)+\rho_{2}\left(g_{n}(X)\right)$. By cash-invariance we may assume that $f_{n}(0)=g_{n}(0)=0$ for all $n \in \mathbb{N}$. Hence, by lemma B.1, there is a subsequence $\left(f_{n_{k}}\right)_{k \in \mathbb{N}}$ of $\left(f_{n}\right)_{n \in \mathbb{N}}$ and a 1-Lipschitz-continuous and increasing function $f: \mathbb{R} \rightarrow \mathbb{R}$ such that $f(a)=\lim _{k \rightarrow \infty} f_{n_{k}}(a)$ for all $a \in \mathbb{R}$. Clearly, the sequence $f_{n_{k}}(X)$ converges $\mathbb{P}$-a.s. to $f(X)$ and $g_{n_{k}}(X)=X-f_{n_{k}}(X)$ converges $\mathbb{P}$-a.s. to $g(X)$ where $g:=\operatorname{Id}_{\mathbb{R}}-f$ is a 1-Lipschitz-continuous increasing function. Since $\left|f_{n_{k}}(X)\right| \leq|X|$ and $\left|g_{n_{k}}(X)\right| \leq|X|$ for all $k \in \mathbb{N}$, we may apply the dominated convergence theorem which yields $f(X), g(X) \in L^{1}$ and $\| f(X)-$ $f_{n_{k}}(X)\left\|_{1} \rightarrow 0,\right\| g(X)-g_{n_{k}}(X) \|_{1} \rightarrow 0$ for $k \rightarrow \infty$. On the one hand, by l.s.c., we have

$$
\begin{aligned}
\rho_{1} \square \rho_{2}(X) & =\lim _{k \rightarrow \infty} \rho_{1}\left(f_{n_{k}}(X)\right)+\rho_{2}\left(g_{n_{k}}(X)\right) \\
& \geq \liminf _{k \rightarrow \infty} \rho_{1}\left(f_{n_{k}}(X)\right)+\liminf _{k \rightarrow \infty} \rho_{2}\left(g_{n_{k}}(X)\right) \\
& \stackrel{\text { l.s.c. }}{\geq} \rho_{1}(f(X))+\rho_{2}(g(X)) .
\end{aligned}
$$

On the other hand, by very definition of the convolution, we have $\rho_{1} \square \rho_{2}(X) \leq$ $\rho_{1}(f(X))+\rho_{2}(g(X))$. Consequently, the comonotone allocation $(f(X), g(X))$ of $X$ is optimal.

Step 4: $\rho_{1} \square \rho_{2}$ is l.s.c. and law-invariant.

Proof. We claim that $\mathcal{A}_{\rho_{1} \square \rho_{2}}$ is closed. To this end, let $\left(X_{n}\right)_{n \in \mathbb{N}} \subset \mathcal{A}_{\rho_{1} \square \rho_{2}}$ be a sequence converging to some $X$ in $L^{1}$. According to step 3 there are $\left(f_{n}, g_{n}\right) \in \mathbb{A}, n \in \mathbb{N}$, such that $0 \geq \rho_{1} \square \rho_{2}\left(X_{n}\right)=\rho_{1}\left(f_{n}\left(X_{n}\right)\right)+\rho_{2}\left(g_{n}\left(X_{n}\right)\right)$. By cash-invariance we may assume that $f_{n}(0)=g_{n}(0)=0$ for all $n \in \mathbb{N}$. Similar to step 3, employing lemma B.1, we find a subsequence $\left(f_{n_{k}}, g_{n_{k}}\right)_{k \in \mathbb{N}}$ 
of $\left(f_{n}, g_{n}\right)_{n \in \mathbb{N}}$ and $(f, g) \in \mathbb{A}$ such that $f_{n_{k}}\left(X_{n_{k}}\right)$ converges to $f(X)$ in $L^{1}$ and $g_{n_{k}}\left(X_{n_{k}}\right)$ converges to $g(X)$ in $L^{1}$. By l.s.c. of $\rho_{1}$ and $\rho_{2}$ we get

$$
\begin{aligned}
\rho_{1} \square \rho_{2}(X) & \leq \rho_{1}(f(X))+\rho_{2}(g(X)) \\
& \leq \liminf _{k \rightarrow \infty} \rho_{1}\left(f_{n_{k}}\left(X_{n_{k}}\right)\right)+\liminf _{k \rightarrow \infty} \rho_{2}\left(g_{n_{k}}\left(X_{n_{k}}\right)\right) \\
& \leq \liminf _{k \rightarrow \infty} \rho_{1}\left(f_{n_{k}}\left(X_{n_{k}}\right)\right)+\rho_{2}\left(g_{n_{k}}\left(X_{n_{k}}\right)\right) \leq 0,
\end{aligned}
$$

and thus $X \in \mathcal{A}_{\rho_{1} \square \rho_{2}}$. Hence, $\mathcal{A}_{\rho_{1} \square \rho_{2}}$ is closed, i.e. $\rho_{1} \square \rho_{2}$ is l.s.c.. The lawinvariance of $\rho_{1} \square \rho_{2}$ follows from lemma 2.2 and the fact that a l.s.c. convex function on $L^{1}$ is law-invariant if and only if its dual is (see (A.13)).

\section{Optimal Risk Sharing under Constraints}

This application is motivated and explained in [14]. Two agents with initial endowments $X_{1}$ and $X_{2}$ in $L^{p}$, assess their individual risk by means of l.s.c. law-invariant convex risk measures $\rho_{1}$ and $\rho_{2}$ on $L^{p}$, respectively. In order to minimize total and individual risk, they redistribute the aggregate endowment $X=X_{1}+X_{2}$ amongst themselves. As is often the case in practice, this redistribution procedure might be subject to some restrictions in the sense that not every risk sharing of $X$ is admissible. We formalize this by defining the set of admissible risk sharings of $X$ as

$$
A_{X}:=\left\{\left(Y_{1}, Y_{2}\right) \in M_{1} \times M_{2} \mid Y_{1}+Y_{2} \leq X\right\}
$$

where $M_{i} \subset L^{p}$ are closed convex law-invariant cash-invariant (that is, $Y \in M_{i}$ implies $Y+a \in M_{i}$ for all $a \in \mathbb{R}$ ) sets such that $X_{i} \in M_{i}, i=1,2$. Note that we allow for "free disposal", i.e. $X-Y_{1}-Y_{2} \geq 0$ for all $\left(Y_{1}, Y_{2}\right) \in A_{X}$. The optimal risk sharing under constraints problem becomes

$$
\inf _{\left(Y_{1}, Y_{2}\right) \in A_{X}} \rho_{1}\left(Y_{1}\right)+\rho_{2}\left(Y_{2}\right) .
$$

In order to solve (7.12), denote $\rho_{i}^{M_{i}}:=\rho_{i}+\delta\left(\cdot \mid M_{i}\right), i=1,2$. Then

$$
\begin{aligned}
(7.12) & =\inf _{Y_{1}, Y_{2} \in L^{p}, Y_{1}+Y_{2} \leq X} \rho_{1}^{M_{1}}\left(Y_{1}\right)+\rho_{2}^{M_{2}}\left(Y_{2}\right) \\
& =\rho_{1}^{M_{1}} \square \rho_{2}^{M_{2}} \square \delta\left(\cdot \mid L_{+}^{p}\right)(X) \\
& =\rho_{1}^{M_{1}} \square \rho_{2}^{M_{2}} \square-\operatorname{essinf}(X) .
\end{aligned}
$$

Note that $\delta\left(\cdot \mid M_{i}\right)$ is proper, l.s.c., law-invariant, and convex. By (A.15) we know that $\delta\left(E[Y] \mid M_{i}\right) \leq \delta\left(Y \mid M_{i}\right)$ for all $Y \in L^{p}$. Hence $Y \in M_{i}$ implies $E[Y] \in M_{i}$, and thus $\mathbb{R} \subset M_{i}$ by cash-invariance. We conclude that $\rho_{1}^{M_{1}}$ and $\rho_{2}^{M_{2}}$ are l.s.c. law-invariant convex cash-invariant functions. Since - essinf is a l.s.c. law-invariant coherent risk measure, we know by corollary 2.7 that $\rho_{1}^{M_{1}} \square \rho_{2}^{M_{2}} \square-$ essinf is a l.s.c. law-invariant convex risk measure, and that this convolution admits a comonotone optimal allocation $\left(Y_{1}, Y_{2}, Y_{3}\right)$ of $X$. If $\rho_{1}^{M_{1}}\left(Y_{1}\right)+\rho_{2}^{M_{1}}\left(Y_{2}\right)-$ 
$\operatorname{essinf}\left(Y_{3}\right)=\infty$, then any admissible risk sharing of $X$ is optimal. Otherwise, if $\rho_{1}^{M_{1}}\left(Y_{1}\right)+\rho_{2}^{M_{1}}\left(Y_{2}\right)-\operatorname{essinf}\left(Y_{3}\right)<\infty$, then we have that $-\operatorname{essinf}\left(Y_{3}\right)<\infty$, and thus $\left(Y_{1}+\operatorname{essinf}\left(Y_{3}\right), Y_{2}\right)$ is a solution of the optimization problem (7.12). We thus have established a substantial improvement of corollary 3.8 in [14]. Note also that $\left(Y_{1}+\operatorname{essinf}\left(Y_{3}\right), Y_{2}\right)=(f(X), g(X))$ for some increasing functions $f, g: \mathbb{R} \rightarrow \mathbb{R}$.

\section{A Law-invariant Convex Functions on $\mathrm{L}^{\mathrm{p}}$}

The proof of theorem 2.5 draws heavily on the following properties, which are proved [18]. Let $F: L^{p} \rightarrow(-\infty, \infty]$ be a proper l.s.c. convex function, then the following conditions are equivalent:

$$
\begin{aligned}
F \text { is law-invariant } & \Leftrightarrow F^{*} \text { is law-invariant } \\
& \Leftrightarrow X \succeq_{c} Y \text { implies } F(X) \leq F(Y) .
\end{aligned}
$$

Consequently, by Jensen's inequality, if $F$ is law-invariant, then

$$
F(E[X \mid \mathcal{G}]) \leq F(X) \text { for all sub- } \sigma \text {-algebras } \mathcal{G} \subset \mathcal{F} .
$$

Now let $\rho: L^{p} \rightarrow(-\infty, \infty]$ be a l.s.c. law-invariant convex cash-invariant function. Then cash-invariance and (A.15) imply that

$$
\rho(X) \geq-E[X]+\rho(0) .
$$

Clearly,

$$
\rho^{*}(-1)=\sup _{X \in L^{p}} E[-X]-\rho(X) \geq-\rho(0) .
$$

However, if $\rho^{*}(-1)>-\rho(0)$, there must be some $Y \in L^{p}$ such that $E[-Y]-$ $\rho(Y)>-\rho(0)$. But this contradicts (A.16). We thus have shown

$$
\rho^{*}(-1)=-\rho(0) .
$$

\section{B An Arzela-Ascoli Type Argument}

The following lemma is needed for the proofs in sections 5 and 6 .

Lemma B.1. Let $f_{n}: \mathbb{R} \rightarrow \mathbb{R}, n \in \mathbb{N}$, be a sequence of increasing 1-Lipschitzcontinuous functions such that $f_{n}(0) \in[-K, K]$ for all $n \in \mathbb{N}$ where $K \geq 0$ is a constant. Then there is a subsequence $\left(f_{n_{k}}\right)_{k \in \mathbb{N}}$ of $\left(f_{n}\right)_{n \in \mathbb{N}}$ and an increasing 1-Lipschitz-continuous function $f: \mathbb{R} \rightarrow \mathbb{R}$ such that $\lim _{k \rightarrow \infty} f_{n_{k}}(x)=f(x)$ for all $x \in \mathbb{R}$.

Proof. The Lipschitz-continuity guarantees that $f_{n}(x) \in[-K, K+x]$ if $x \geq 0$ and $f_{n}(x) \in[-K+x, K]$ if $x \leq 0$. Hence, by a procedure well-known from the standard proof of the Arzela-Ascoli theorem, we are able to extract a subsequence $\left(f_{n_{k}}\right)_{k \in \mathbb{N}}$ of $\left(f_{n}\right)_{n \in \mathbb{N}}$ such that $\lim _{k \rightarrow \infty} f_{n_{k}}(q)$ exists for all $q \in \mathbb{Q}$. In 
fact, we can easily show that the sequences $\left(f_{n_{k}}(x)\right)_{k \in \mathbb{N}}$ must converge for all $x \in \mathbb{R}$. To this end, let $\epsilon>0$ be arbitrary and choose $q \in \mathbb{Q}$ and $N_{0} \in \mathbb{N}$ such that $|q-x|<\epsilon / 3$ and $\left|f_{n_{k}}(q)-f_{n_{l}}(q)\right|<\epsilon / 3$ for all $k, l \geq N_{0}$. Then for all $k, l \geq N_{0}$ :

$$
\begin{aligned}
\left|f_{n_{k}}(x)-f_{n_{l}}(x)\right| \leq\left|f_{n_{k}}(x)-f_{n_{k}}(q)\right|+\left|f_{n_{k}}(q)-f_{n_{l}}(q)\right|+ \\
+\left|f_{n_{l}}(q)-f_{n_{l}}(x)\right| \\
\leq 2|x-q|+\left|f_{n_{k}}(q)-f_{n_{l}}(q)\right|<\epsilon,
\end{aligned}
$$

in which we did apply the Lipschitz-continuity twice. Now it is easily verified that $f(x):=\lim _{k \rightarrow \infty} f_{n_{k}}(x), x \in \mathbb{R}$, is a 1-Lipschitz-continuous increasing function.

\section{References}

[1] Acciaio, B. (2007), Optimal Risk Sharing with Non-monotone Monetary Functionals, Finance and Stochastics, 11, 267-289.

[2] Aliprantis, C. and Border, K. (1999), Infinite Dimensional Analysis, Springer.

[3] Artzner, P., Delbaen, F., Eber, J.M. and Heath, D. (1999), Coherent Measures of Risk, Mathematical Finance, 9, 203-228.

[4] Barrieu, P. and El Karoui, N. (2005), Inf-convolution of Risk Measures and Optimal Risk Transfer, Finance and Stochastics, Vol. 9, nb. 2, 269-298.

[5] Barrieu, P. and El Karoui, N. (2004), Optimal Derivatives Design under Dynamic Risk Measures, Yin, G. and Zhang, Q. (eds.): Mathematics of Finance. Contemporary Mathematics - A.M.S., 13-25.

[6] Biagini, S. and Frittelli, M. (2006), On Continuity Properties and Dual Representation of Convex and Monotone Functinals on Frechet Lattices, Working Paper.

[7] Burgert, C. and Rüschendorf, L. (2005), Allocations of Risk and Equilibrium in Markets with Finitely Many Traders, preprint.

[8] Cheridito, P. and Li, T. (2007), Risk measures on Orlicz hearts, forthcoming in Mathematical Finance.

[9] Cherny, A. (2007), Capital Allocation and Risk Contribution with Discretetime Coherent Risk, preprint.

[10] Cherny, A. and Kupper, M. (2007), Divergence Utilities, VIF Working Paper.

[11] Dana, R.-A. and Scarsini, M. (2004), Optimal Risk Sharing with Background Risk, preprint. 
[12] Delbaen, F. (2002), Coherent Risk Measures on General Probability Spaces, Advances in Finance and Stochastics, 1-37, Springer.

[13] Ekeland, I. and Témam, R. (1999), Convex Analysis and Variational Problems, Siam.

[14] Filipović, D. and Kupper, M. (2006), Equilibrium Prices for Monetary Utility Functions, International Journal of Theoretical and Applied Finance, forthcoming.

[15] Filipović, D. and Kupper, M. (2007), Optimal Capital and Risk Transfers for Group Diversification, Mathematical Finance, 18, 55-76.

[16] Filipović, D. and Kupper, M. (2007), On the Group Level Swiss Solvency Test, Mitteilungen der Schweizer Aktuarvereinigung, 1.

[17] Filipović, D. and Kupper, M. (2007), Monotone and Cash-Invariant Convex Functions and Hulls, Insurance: Mathematics and Economics, 41, 1-16.

[18] Filipović, D. and Svindland, G. (2007), Convex Risk Measures Beyond Bounded Risks, or The Canonical Model Space for Law-Invariant Risk Measures is $L^{1}$, VIF Working Paper.

[19] Föllmer, H. and Schied, A. (2002), Stochastic Finance, An Introduction in Discrete Time, de Gruyter Studies in Mathematics 27.

[20] Fritelli, M. and Rosazza Gianin, E. (2005), Law-invariant Convex Risk Measures, Advances in Mathematical Economics, 7, 33-46.

[21] Jouini, E., Schachermayer, W. and Touzi, N. (2005), Optimal Risk Sharing for Law-Invariant Monetary Utility Functions, Mathematical Finance, forthcoming.

[22] Jouini, E., Schachermayer, W. and Touzi, N. (2006), Law-Invariant Risk Measures have the Fatou Property, Advances in Mathematical Economics, Vol. 9, 49-71.

[23] Kaina, M. and Rüschendorf, L. (2007), On Convex Risk Measures on $L^{p}$, Working Paper.

[24] Ludkovski, M. and Rüschendorf, L. (2007), On comonotonicity of Pareto optimal risk sharing, Statistics and Probability Letters, forthcoming.

[25] Landsberger, M. and Meilijson, I. (1994), Comonotone allocations, BickelLehmann dispersion and the Arrow-Pratt measure of risk aversion, Annals of Operations Research, 52.

[26] Rockafellar, R.T. (1997), Convex Analysis, Princeton University Press. 